\title{
A THEOREM ON CONTINUED FRACTIONS AND THE FUNDAMENTAL INEQUALITIES
}

\author{
DAVID F. DAWSON
}

Scott and Wall [3] showed that if the inequalities

$$
\begin{gathered}
r_{1}\left|1+a_{1}\right| \geqq\left|a_{1}\right|, \quad r_{2}\left|1+a_{1}+a_{2}\right| \geqq\left|a_{2}\right|, \\
r_{p}\left|1+a_{p-1}+a_{p}\right| \geqq r_{p} r_{p-2}\left|a_{p-1}\right|+\left|a_{p}\right|, \quad p=3,4,5, \cdots,
\end{gathered}
$$

hold and lim inf $r_{1} r_{2} \cdots r_{p}=0$, then the continued fraction

$$
f(a)=\frac{1}{1}+\frac{a_{1}}{1}+\frac{a_{2}}{1}+\frac{a_{3}}{1}+\cdots
$$

converges at least in the wider sense, and if actual inequality holds in either of the first two relations of (1), then $f(a)$ converges. The $r_{p}$ are nonnegative numbers and the $a_{p}$ are complex numbers. In this note the following theorem is established.

Theorem A. If the inequalities (1) hold, $r_{p_{i}}<1, i=1,2,3, \cdots$, and $\sum\left(1-r_{p_{i}}\right)$ diverges, then $f(a)$ converges at least in the wider sense, and if actual inequality holds in either of the first two relations of (1), then $f(a)$ converges.

Clearly the condition of Scott and Wall on the $r_{p}$ implies the condition of Theorem A, but not conversely. Theorem A is an immediate consequence of the following result.

Theorem B. If the inequalities (1) hold for $r_{p}$ of even index, no $a_{2 p}=0, r_{2 p_{i}}<1, i=1,2,3, \cdots, \sum\left(1-r_{2 p_{i}}\right)$ diverges, and $v$ is the point in the extended complex plane to which the odd part of $f(a)$ converges at least in the wider sense, then every neighborhood of $v$ contains infinitely many even approximants of $f(a)$. A similar result holds if the roles of even and odd indices are interchanged.

Proof. Let $f_{p}=A_{p} / B_{p}$ denote the $p$ th approximant of $f(a)$. Consider first the case that some $a_{2 p-1}=0$. If for some positive integer $i$, $B_{2 i}=0$ and $B_{2 i+2}=0$, then by the fundamental recurrence formulas for $f(a), B_{2 i+1}=0$. But since $r_{2 p}\left|B_{2 p+1}\right| \geqq\left|a_{2 p}\right|\left|B_{2 p-1}\right|, p=1,2,3, \cdots$, no $B_{2 p+1}=0$. Hence $B_{p} B_{p+1} \neq 0$ for infinitely many values of $p$. Thus the theorem holds for this case since

Presented to the Society, April 14, 1961; received by the editors June 19, 1961 and, in revised form, August 2, 1961. 


$$
\left|f_{p+1}-f_{p}\right|=\left|a_{1} a_{2} \cdots a_{p}\right| /\left|B_{p+1} B_{p}\right| \quad \text { if } B_{p} B_{p+1} \neq 0,
$$

and

$$
\left|f_{2 p+1}-f_{2 p-1}\right|=\left|a_{1} a_{2} \cdots a_{2 p-1}\right| /\left|B_{2 p+1} B_{2 p-1}\right|, \quad p=1,2,3, \cdots \text {. }
$$

Suppose no $a_{2 p-1}=0$. Since the inequalities (1) hold for $r_{p}$ of even index and no $a_{2 p}=0$, then no $r_{2 p}=0$ and $f(a)$ converges absolutely at least in the wider sense $[2$, p. $137 ; 3]$. By Lemma 2.2 of [1], therefore, either the odd part of $f(a)$ converges absolutely or else the reciprocal of the odd part of $f(a)$ converges absolutely to 0 . Suppose the odd part of $f(a)$ converges absolutely. Let $q$ be a term of the sequence $\left\{p_{i}\right\}$. Then

$$
\begin{aligned}
\left|f_{2 q+1}-f_{2 q}\right| & =\frac{\left|a_{1} a_{2} \cdots a_{2 q}\right|}{\left|B_{2 q+1} B_{2 q}\right|}=\frac{\left|a_{1} a_{2} \cdots a_{2 q-1}\right|}{\left|B_{2 q+1} B_{2 q-1}\right|} \cdot \frac{\left|a_{2 q} B_{2 q-1}\right|}{\left|B_{2 q}\right|} \\
& \leqq\left|f_{2 q+1}-f_{2 q-1}\right| \cdot \frac{\left|a_{2 q} B_{2 q-1}\right|}{\left|B_{2 q+1}\right|-\left|a_{2 q} B_{2 q-1}\right|} \\
& \leqq\left|f_{2 q+1}-f_{2 q-1}\right| \cdot \frac{r_{2 q}\left|B_{2 q+1}\right|}{\left|B_{2 q+1}\right|-r_{2 q}\left|B_{2 q+1}\right|} \\
& \leqq \frac{\left|f_{2 q+1}-f_{2 q-1}\right|}{1-r_{2 q}} .
\end{aligned}
$$

Thus $\lim \inf \left|f_{2 p_{i}+1}-f_{2 p_{i}}\right|=0$ since $\sum\left|f_{2 p+1}-f_{2 p-1}\right|$ converges and $\sum\left(1-r_{2 p_{i}}\right)$ diverges. Hence the theorem holds for this case.

Suppose next that the reciprocal of the odd part of $f(a)$ converges absolutely to zero. This means that the even part of the continued fraction

$$
\frac{1}{1}+\frac{a_{2}}{1}+\frac{a_{3}}{1}+\frac{a_{4}}{1}+\cdots
$$

converges absolutely to $-1 / a_{1}$. Suppose some neighborhood of the point $-1 / a_{1}$ does not contain infinitely many odd approximants of (2). Let $a_{1}^{\prime} \neq 0$ be chosen in such a way that $r_{2}\left|1+a_{1}^{\prime}+a_{2}\right|>\left|a_{2}\right|$. Then the odd part of the continued fraction

$$
\frac{1}{1}+\frac{a_{1}^{\prime}}{1}+\frac{a_{2}}{1}+\frac{a_{3}}{1}+\cdots
$$

converges absolutely to $1 /\left(1-a_{1}^{\prime} / a_{1}\right)$. By the part of this theorem which was proved above, every neighborhood of $1 /\left(1-a_{1}^{\prime} / a_{1}\right)$ contains infinitely many even approximants of (3). But this contradicts 
the assumption that some neighborhood of $-1 / a_{1}$ does not contain infinitely many odd approximants of (2). Thus every neighborhood of $\infty$ contains infinitely many even approximants of $f(a)$, and the theorem holds for this case.

The proof of the theorem for the case that the roles of odd and even indices are interchanged is similar and will be omitted.

The following corollary is obvious.

CoROLlaRY. If the phrase " $r_{2 p_{i}}<1, i=1,2,3, \cdots$, and $\sum\left(1-r_{2 p_{i}}\right)$ diverges" in Theorem B is replaced with "lim inf $r_{2} r_{4} \cdots r_{2 p}=0$," then the conclusion of Theorem B holds.

The method of proof used in proving Theorem B for the case that $v=\infty$ can be used to extend Theorem 1 of [4] and Theorem 3.1 of [1].

THEOREM C. If the odd (even) part of the partially bounded continued fraction $f(a)$ converges at least in the wider sense to a point $v$ in the extended complex plane, then every neighborhood of $v$ contains infinitely many even (odd) approximants of $f(a)$.

Proof. Consider the case that the odd part of $f(a)$ converges at least in the wider sense to $v$, where $v$ is finite or $\infty$. In case some $a_{p}=0$ and $B_{q} B_{q+1} \neq 0$ for infinitely many values of $q$, the theorem follows immediately. Suppose some $a_{p}=0$ and $B_{q} B_{q+1}=0$ for all but a finite number of values of $q$. Then by the fundamental recurrence formulas for $f(a)$, there exists a positive integer $i$ such that if $q>i$, then $B_{q}=0$. Thus there exists $j$ such that if $p>j$, then $A_{2 p+1} \neq 0$. Therefore $A_{2 p} \neq 0$ for infinitely many values of $p$, and the theorem follows for this case. Next consider the case that no $a_{p}=0$. Suppose some neighborhood of $v$ does not contain infinitely many even approximants of $f(a)$. This means that the even part of the continued fraction (2) converges at least in the wider sense to a point $v^{\prime}$, where $v^{\prime}$ is either finite or $\infty$, and there exists a neighborhood $N$ of $v^{\prime}$ which does not contain infinitely many odd approximants of (2). Let $a_{1}^{\prime} \neq 0$ be a point such that $-1 / a_{1}^{\prime}$ is in $N$ but is distinct from $v^{\prime}$. Let $r$ be a positive number such that if $\left|u-\left(-1 / a_{1}^{\prime}\right)\right| \leqq r$, then $u$ is in $N$. Since the linear fractional transformation $T(u)=1 /\left(1+a_{1}^{\prime} u\right)$ takes the exterior of the circle $\left|u+1 / a_{1}^{\prime}\right|=r$ to the interior of the circle $|z|=1 / r\left|a_{1}^{\prime}\right|$, it follows that all but at most a finite number of even approximants of (3) lie in the circle $|z|=1 / r\left|a_{1}^{\prime}\right|$. Hence the continued fraction (3) is partially bounded, the odd part of (3) converges to $1 /\left(1+a_{1}^{\prime} \cdot v^{\prime}\right)$, there exist a number $M$ and a positive integer $k$ such that if $f_{p}^{\prime}$ is the $p$ th approximant of (3) and $p>k$, then $\left|f_{p}^{\prime}\right|<M$, and no infinite subsequence of the sequence of even approximants of 
(3) converges to $1 /\left(1+a_{1}^{\prime} \cdot v^{\prime}\right)$. Therefore by Theorem 1 of [4] the assumption that some neighborhood of $v$ does not contain infinitely many even approximants of $f(a)$ is false. Hence the theorem is proved for the case considered here. The other case is similar.

\section{REFERENCES}

1. D. F. Dawson, Concerning convergence of continued fractions, Proc. Amer. Math. Soc. 11 (1960), 640-647.

2. - Continued fractions with absolutely convergent even or odd part, Canad. J. Math. 11 (1959), 131-140.

3. W. T. Scott and H. S. Wall, A convergence theorem for continued fractions, Trans. Amer. Math. Soc. 47 (1940), 155-172.

4. H. S. Wall, Partially bounded continued fractions, Proc. Amer. Math. Soc. 7 (1956), 1090-1093.

North Texas State University

\section{A CHARACTERIZATION OF QF-3 ALGEBRAS}

\section{HIROYUKI TACHIKAWA}

Let $A$ be an associative algebra with a unit element 1 and

$$
0 \rightarrow A \rightarrow X_{1} \rightarrow X_{2} \rightarrow \cdots \rightarrow X_{n}
$$

an exact sequence of $A$ - $A$-homomorphisms with $A$ - $A$-projective modules $X_{p}, 1 \leqq p \leqq n$. Recently concerning complete homology of algebras, Nakayama [4] has proposed to classify algebras according to how long an exact sequence (1) they have. In this paper we shall show that the first class in his classification is the class of QF-3 algebras (for definition see Thrall [5]), that is to say, $A$ is QF-3 if and only if $A$ has an exact sequence $0 \rightarrow A \rightarrow X$, where $X$ is $A$ - $A$-projective.

To begin with we state

LEMma 1. $A$ is a QF-3 algebra if and only if $A$ has a faithful left $A$-module which is projective and injective.

This lemma was already used in [2] and [3] and for the proof we shall refer to Theorems 3.1 and 3.2 of [1].

Now we shall prove

Received by the editors August 14, 1961. 